\title{
Exosomal circRNAs: new players in the field of cholangiocarcinoma
}

\author{
Corentin Louis ${ }^{1}$, Matthis Desoteux ${ }^{2}$ and Cédric Coulouarn ${ }^{3, *}$ \\ 1 Inserm, Univ Rennes, France; corentin.louis@univ-rennes1.fr \\ 2 Inserm, Univ Rennes, France; matthis.desoteux@univ-rennes1.fr \\ 3 Inserm, Univ Rennes, France; cedric.coulouarn@inserm.fr
}

* Correspondence: Inserm U1241, CHU Pontchaillou, 2 rue Henri Le Guilloux, 35033

Rennes, France ; cedric.coulouarn@inserm.fr; Tel.: +33-223-233-881

\section{Abstract}

Cholangiocarcinoma (CCA) is a deadly cancer worldwide associated with limited therapeutic options. A recent study published in Clinical Science by Wang and colleagues [Clinical Science (2019) 133, 1935-1953] brought new perspectives to CCA management and therapy by focusing on circular RNAs (circRNAs). CircRNAs belong to an emerging class of functional non-coding RNAs (ncRNAs) regulating numerous biological processes. Notably, circRNAs have been associated with cancer onset and progression, although reports in CCA are very limited so far. In this work, the expression of circular RNA circ-0000284 (aka circHIPK3) was specifically elevated in CCA cell lines, human tumor tissues and plasma exosomes. Gain and loss of function approaches were performed to better understand the molecular mechanisms regulated by circ-0000284. Notably, the authors evaluated the role of circ-0000284 as a microRNA (miRNA) sponge. By prediction analysis and functional tests, a direct interaction was demonstrated with miR-637 that targets lymphocyte antigen-6 E (LY6E). Increased expression of circ-0000284 was associated with enhanced migration, invasion and proliferation of CCA cell lines. Interestingly, exosomal-mediated circ-0000284 was reported to exhibit pro-oncogenic effects on surrounding normal cells. Altogether, these 
data not only highlight circRNAs as new players in CCA pathogenesis but also as promising molecules for innovative non-invasive biomarkers, as circRNAs are enriched and stable in exosomes. Further investigations on extracellular vesicles should provide the necessary tools to improve CCA diagnosis, and move towards targeted-therapies.

Keywords: Cholangiocarcinoma, circular RNA, exosomes, biomarkers

\section{Comment on}

Circ-0000284 arouses malignant phenotype of cholangiocarcinoma cells and regulates the biological functions of peripheral cells through cellular communication. [Clin Sci (Lond). 2019] 


\section{Introduction}

Cholangiocarcinoma (CCA) refer to a heterogeneous group of aggressive tumors that arise within both intrahepatic and extrahepatic bile ducts. CCA represent the second most common cancer in the liver after hepatocellular carcinoma (HCC). CCA incidence has been steadily increasing during the last decades and the absence of characteristic symptoms restrains early diagnosis (1). In addition, resistance of CCA to conventional chemotherapies and radiotherapies approaches limits therapeutic options (2). Surgical approaches remain the best possibly curative alternative for a total recovery. However, even total resections with safety margins are associated with a high rate of recurrence and a 5-year survival ranging from $15 \%$ to $40 \%$ (3). Hence, fresh ideas aiming at identifying novel therapeutic strategies and biomarkers are urgently needed to improve the survival of patients with CCA.

\section{circRNAs: an emerging class of functional ncRNAs}

Recently, circular RNAs (circRNAs) emerged in the literature as a puzzling class of noncoding RNAs (ncRNAs). For a long time, circRNAs were viewed as splicing noise or byproducts of splicing errors. Eventually, they drew increasing interest after being found dysregulated in several types of cancer, including CCA (4). Subsequently, growing evidences indicated that circRNAs play a key role in cancer onset and progression, by regulating notably cell proliferation, migration or invasion (5). It is now recognized that circRNAs, unlike linear RNAs, are generated through back-splicing and form a covalently closed continuous loop without 3' and 5' extremities. This specific structure renders circRNAs more stable and resistant to exonuclease degradation. Hence, circRNAs could be of a valuable assistance for clinical diagnosis, possibly serving as potent predictive non-invasive biomarkers, notably in CCA (6). The origin, biogenesis, and functions of circRNAs remained unclear for many years. Nevertheless, points of consensus are starting to emerge in the literature. For instance, one of the latest studies in the field strengthened the established process governing circRNA biogenesis (7). Thus, it was confirmed that circRNAs are natural byproducts of spliceosomemediated splicing. Notably, a model whereby intron definition, exon definition and back- 
splicing require the same spliceosome machinery has been highlighted, suggesting that alternative back-splicing may compete with canonical splicing and thus regulate cell function at a post-transcriptional level. Interestingly, canonical and alternative back-splicing may be regulated by cis or trans factors (7). Accordingly, Conn et al. identified the TGF $\beta$-induced RNA binding protein Quaking (QKI) as a regulator of circRNA biogenesis binding on introns flanking circRNA-forming exons (8).

Almost 30 years after the discovery of the first spliced circular products (9), active research in the field improved our understanding of circRNA function. Thus, circRNAs were mainly described as post-transcriptional regulators due to their capability to sponge microRNAs (miRNAs). However, additional functions have been attributed to circRNAs over the last years, including sequestration of proteins, competition with canonical mRNA splicing, and transcriptional regulation of their host gene (10). In addition, a new school of thought is reconsidering the non-coding nature of some circRNAs. This assertion is supported by recent evidences identifying translation initiation systems in circRNAs (11).

\section{circRNAs: a wide-open field for research in CCA}

To date, only few recent studies investigated the role of circRNAs in CCA pathogenesis. One of them focused on CDR1AS, a circRNA already largely described for its implication in cancer onset and progression. Thus, CDR1AS expression was significantly higher in CCA tissue samples and correlated with clinical features such as lymph node invasion, advanced TNM stage, and a poor overall survival (12). Another study reported the expression of circRNAs in intrahepatic cholangiocarcinoma (iCCA). Gene expression profiling using pangenomic microarrays identified TGF $\beta$-induced long ncRNA TLINC (formerly known as CASC15) in iCCA cell lines. Subsequently, this work revealed the presence of circular isoforms of TLINC in iCCA tissue samples (13). Although the function of TLINC circRNA in CCA carcinogenesis was not explicitly explored, a putative role in cancer stem cell biology is supported by an elevated expression of TLINC circRNA in human embryonic stem cells. (14). A couple of studies performed by $\mathrm{Xu}$ et al. pinpointed two aberrantly expressed circRNAs in 
both CCA cell lines and human tumors. The involvement of these circRNAs in CCA progression has been determined by both in vitro and in vivo experiments. Thus, circ0001649 and circ-0005230 were respectively down- and up-regulated in CCA cell lines and human tumors and their role as putative miRNA sponge was evaluated. In both cases, these circRNAs were associated with CCA progression and deserve further investigations as therapeutic targets for $\operatorname{CCA}(4,15)$. In fact, most of the studies reported in CCA so far highlighted circRNA deregulations and their clinical relevance rather than performing a deep and comprehensive functional analysis.

\section{A comprehensive analysis of circ-0000284 (circHIPK3) in CCA}

The recent study published in Clinical Science by Wang and colleagues identified for the first time in CCA, circ-0000284, an isoform of the well-known circHIPK3 circRNA involved in tumor progression (16). Prediction data reported 21 isoforms of circHIPK3 deriving from the same pre-mRNA (17). Although all these isoforms were extensively described in cancer, circ0000284 is definitely the most investigated one (18-24). However, opposite actions for circ0000284 were reported depending on the type of cancer (Table 1). Thus, circ-0000284 can exhibit either an oncogenic or a tumor suppressor role. As it stands, it is impossible to conclude firmly on a general function of circ-0000284 in cancer. However, it seems increasingly clear that circ-0000284 acts mainly as a miRNA sponge (Table 1). To this extent, there are strong evidences showing that circ-0000284 rather exhibits pro-oncogenic features. Further investigations are needed to determine whether specific circHIPK3 isoforms carry either oncogenic or tumor-suppressive features. As far as CCA is concerned, the recent work by Wang and colleagues demonstrated that circ-0000284 was able to sponge miR-637 and to promote CCA progression. Hence, the authors demonstrated that circ0000284 acts as an oncogene by promoting CCA cell migration, invasion and proliferation. Further mechanistic experiments established the ability of circ-0000284 to sponge miR-637, leading to increased expression of lymphocyte antigen-6 E (LY6E) through a competitive binding to miR-637 (Figure 1). LY6 complex is a group of alloantigens that was first 
discovered on lymphocytes. Interestingly, increased expression of LY6 family was reported in multiple types of cancer, and was subsequently associated with poor overall and disease free survival. Thus, high LY6E expression was significantly correlated with poor clinical outcome in colorectal cancer, glioma, ovarian cancer, gastric cancer, breast cancer and lung cancer. Altogether, these data suggest that LY6 family members play a crucial role in tumor progression and represent clinically relevant target (25). To date, these data are the first to support a correlation between a high LY6E expression and a pro-oncogenic action in CCA, and providing a noteworthy mechanistic explanation (16). However, LY6E is not the only target of miR-637 as reported in the literature (Table 2). Thus, miR-637 could affect CCA progression by targeting additional transcripts encoding proteins involved in cell proliferation and migration (e.g. CDK6, STAT3, AKT1) (Table 2). In addition, miR-637 could be itself regulated by non-coding endogenous competitors other than solely circHIPK3, as described in other diseases (26-34) (Table 2).

\section{Exosomal circRNAs as non-invasive functional biomarkers in CCA}

An important aspect of the study by Wang and colleagues relies on evaluating for the first time in CCA the influence of exosomal-transmited circRNAs on their immediate cellular microenvironment. Hence, autocrine and paracrine pro-tumorigenic actions mediated by exosomes-carried circRNAs (exo-circRNAs) were demonstrated (Figure 1). Although the elevated expression of exo-circ-0000284 has been measured in the host surrounding cells, no further information is provided as regard to the mechanisms that promotes cell migration and cell proliferation in these host cells. It was assumed that transformation of host cells was mediated by the same mechanism than the one described in tumor cells where exo-circ0000284 was originating from. Thus, a mechanism involving miR-637 sponging needs to be more clearly defined. In addition, the possibility that exo-circ-0000284 involves different miRNA regulatory networks in host cells should not be excluded. Interestingly, an increased expression of circ-0000284 was also shown in plasma-derived exosomes from patients with CCA. To our knowledge, this is the first report of circulating exo-circRNAs in CCA. Thus, 
these findings provided a promising alternative for existing CCA biomarkers. Similarly, a recent paper highlighted the clinical relevance of protein biomarkers conveyed in body fluids via extracellular vesicles (EVs) in CCA (35). Thus, CCA-derived EVs from patient serum might serve as cargo for oncogenic proteins (35). Similar to circRNAs, EV-derived biomarkers are about to progressively challenge the poor established biomarkers in CCA. For instance, serum level of carbohydrate antigen 19-9 (CA19-9) or carcinoembryonic antigen (CEA) are two possible biomarkers to suspect CCA diagnosis. However, several studies reported the poor specificity and sensitivity of these biomarkers, highlighting the need for more efficient substitutes for CCA diagnosis and prognosis (36-38). For that purpose, it would be of great interest to identify clinically relevant circRNA signatures in CCA to improve patient outcome, as circRNAs are easily detectable and stable in body fluids.

\section{Conclusion}

In conclusion, recent data provide evidence of circRNAs potential as robust biomarkers with diagnostic and prognostic values in CCA. Remarkably, Wang and colleagues identified, for the first time in CCA, an exosome-mediated circRNA that was able to display autocrine and paracrine actions promoting CCA progression. Interestingly, this circRNA was also found in plasma-derived-exosomes, opening new perspectives for the use of exo-circRNAs as noninvasive biomarkers. Further investigations should provide the necessary tools to improve CCA diagnosis, and move towards targeted-therapies.

\section{Acknowledgements}

\section{Financial support and sponsorship}

This work was supported by Inserm, Université de Rennes 1, INCa, and ITMO Cancer AVIESAN (Alliance Nationale pour les Sciences de la Vie et de la Santé) dans le cadre du Plan cancer (Non-coding RNA in cancerology: fundamental to translational; [Grant C18007NS to C.C.]). C.L. is supported by a PhD fellowship from Université de Rennes 1. 
M.D. is supported by a PhD fellowship from Ligue Contre le Cancer (CD22) and Région Bretagne. The authors are members of the European Network for the Study of Cholangiocarcinoma (ENSCCA) and participate in the initiative COST Action EUROCHOLANGIO-NET granted by the COST Association (CA18122).

\section{Conflicts of interest}

None.

Abbreviations: CCA, cholangiocarcinoma; circRNAs, circular RNAs; EVs, extracellular vesicles; exo-circRNAs, exosomes circRNAs; HCC, hepatocellular carcinoma; LY6E, lymphocyte antigen-6 E; miRNAs, microRNAs; ncRNAs, non-coding RNAs.

\section{Figure legend:}

Figure 1: Circ-0000284 promotes tumor progression in CCA cells acting as competitive endogenous RNA by sponging miR-637. Circ-0000284 holds sway over surrounding cells biological functions through exosomal communication. Exosomes released in blood represent a potential biomarker for CCA.

(modified from Servier Medical images: https://smart.servier.com).

\section{References}

1. Bertuccio P, Malvezzi M, Carioli G, Hashim D, Boffetta P, El-Serag HB, et al. Global trends in mortality from intrahepatic and extrahepatic cholangiocarcinoma. J Hepatol. 2019;71(1):104-14.

2. Rizvi S, Khan SA, Hallemeier CL, Kelley RK, Gores GJ. Cholangiocarcinoma - evolving concepts and therapeutic strategies. Nat Rev Clin Oncol. 2018;15(2):95-111.

3. Bridgewater J, Galle PR, Khan SA, Llovet JM, Park JW, Patel T, et al. Guidelines for the diagnosis and management of intrahepatic cholangiocarcinoma. J Hepatol. 2014;60(6):1268-89.

4. Xu Y, Yao Y, Zhong X, Leng K, Qin W, Qu L, et al. Downregulated circular RNA hsa_circ_0001649 regulates proliferation, migration and invasion in cholangiocarcinoma cells. Biochem Biophys Res Commun. 2018;496(2):455-61.

5. Bach DH, Lee SK, Sood AK. Circular RNAs in Cancer. Mol Ther Nucleic Acids. 2019;16:118-29.

6. Li Y, Zheng Q, Bao C, Li S, Guo W, Zhao J, et al. Circular RNA is enriched and stable in exosomes: a promising biomarker for cancer diagnosis. Cell Res. 2015;25(8):981-4.

7. Li X, Liu S, Zhang L, Issaian A, Hill RC, Espinosa S, et al. A unified mechanism for intron and exon definition and back-splicing. Nature. 2019;573(7774):375-80.

8. Conn SJ, Pillman KA, Toubia J, Conn VM, Salmanidis M, Phillips CA, et al. The RNA binding protein quaking regulates formation of circRNAs. Cell. 2015;160(6):1125-34. 
9. Nigro JM, Cho KR, Fearon ER, Kern SE, Ruppert JM, Oliner JD, et al. Scrambled exons. Cell. 1991;64(3):607-13.

10. Barrett SP, Salzman J. Circular RNAs: analysis, expression and potential functions. Development. 2016;143(11):1838-47.

11. Schneider T, Bindereif A. Circular RNAs: Coding or noncoding? Cell Res. 2017;27(6):724-5.

12. Jiang XM, Li ZL, Li JL, Xu Y, Leng KM, Cui YF, et al. A novel prognostic biomarker for cholangiocarcinoma: circRNA Cdr1as. Eur Rev Med Pharmacol Sci. 2018;22(2):365-71.

13. Merdrignac A, Angenard G, Allain C, Petitjean K, Bergeat D, Bellaud P, et al. A novel transforming growth factor beta-induced long noncoding RNA promotes an inflammatory microenvironment in human intrahepatic cholangiocarcinoma. Hepatol Commun. 2018;2(3):254-69.

14. Salzman J, Chen RE, Olsen MN, Wang PL, Brown PO. Cell-type specific features of circular RNA expression. PLoS Genet. 2013;9(9):e1003777.

15. Xu Y, Yao Y, Liu Y, Wang Z, Hu Z, Su Z, et al. Elevation of circular RNA circ_0005230 facilitates cell growth and metastasis via sponging miR-1238 and miR-1299 in cholangiocarcinoma. Aging (Albany NY). 2019;11(7):1907-17.

16. Wang S, Hu Y, Lv X, Li B, Gu D, Li Y, et al. Circ-0000284 arouses malignant phenotype of cholangiocarcinoma cells and regulates the biological functions of peripheral cells through cellular communication. Clin Sci (Lond). 2019;133(18):1935-53.

17. Zhong $\mathrm{S}$, Wang J, Zhang $\mathrm{Q}, \mathrm{Xu} \mathrm{H}$, Feng J. CircPrimer: a software for annotating circRNAs and determining the specificity of circRNA primers. BMC Bioinformatics. 2018;19(1):292.

18. Jeck WR, Sorrentino JA, Wang K, Slevin MK, Burd CE, Liu J, et al. Circular RNAs are abundant, conserved, and associated with ALU repeats. RNA. 2013;19(2):141-57.

19. Zheng Q, Bao C, Guo W, Li S, Chen J, Chen B, et al. Circular RNA profiling reveals an abundant circHIPK3 that regulates cell growth by sponging multiple miRNAs. Nat Commun. 2016;7:11215.

20. Chen $G$, Shi Y, Liu M, Sun J. circHIPK3 regulates cell proliferation and migration by sponging miR-124 and regulating AQP3 expression in hepatocellular carcinoma. Cell Death Dis. 2018;9(2):175. 21. Jin $P$, Huang $Y$, Zhu P, Zou Y, Shao T, Wang O. CircRNA circHIPK3 serves as a prognostic marker to promote glioma progression by regulating miR-654/IGF2BP3 signaling. Biochem Biophys Res Commun. 2018;503(3):1570-4.

22. Ke Z, Xie F, Zheng C, Chen D. CircHIPK3 promotes proliferation and invasion in nasopharyngeal carcinoma by abrogating miR-4288-induced ELF3 inhibition. J Cell Physiol. 2019;234(2):1699-706.

23. Li Y, Zheng F, Xiao X, Xie F, Tao D, Huang C, et al. CircHIPK3 sponges miR-558 to suppress heparanase expression in bladder cancer cells. EMBO Rep. 2017;18(9):1646-59.

24. Zeng K, Chen X, Xu M, Liu X, Hu X, Xu T, et al. CircHIPK3 promotes colorectal cancer growth and metastasis by sponging miR-7. Cell Death Dis. 2018;9(4):417.

25. Luo L, McGarvey P, Madhavan S, Kumar R, Gusev Y, Upadhyay G. Distinct lymphocyte antigens 6 (Ly6) family members Ly6D, Ly6E, Ly6K and Ly6H drive tumorigenesis and clinical outcome. Oncotarget. 2016;7(10):11165-93.

26. Zhang $Q$, Wang G, Xu L, Yao Z, Song L. Long non-coding RNA LINC00473 promotes glioma cells proliferation and invasion by impairing miR-637/CDK6 axis. Artif Cells Nanomed Biotechnol. 2019;47(1):3896-903.

27. Zhang M, Xia B, Xu Y, Zhang Y, Xu J, Lou G. Circular RNA (hsa_circ_0051240) promotes cell proliferation, migration and invasion in ovarian cancer through miR-637/KLK4 axis. Artif Cells Nanomed Biotechnol. 2019;47(1):1224-33.

28. Rui $X, X u Y$, Jiang $X$, Ye W, Huang $Y$, Jiang J. Long non-coding RNA C5orf66-AS1 promotes cell proliferation in cervical cancer by targeting miR-637/RING1 axis. Cell Death Dis. 2018;9(12):1175.

29. Zhang Y, Li C, Liu X, Wang Y, Zhao R, Yang Y, et al. circHIPK3 promotes oxaliplatin-resistance in colorectal cancer through autophagy by sponging miR-637. EBioMedicine. 2019.

30. Wang L, Jiang F, Xia X, Zhang B. LncRNA FAL1 promotes carcinogenesis by regulation of miR637/NUPR1 pathway in colorectal cancer. Int J Biochem Cell Biol. 2019;106:46-56. 
31. Huang W, Cao J, Peng X. LINC01234 facilitates growth and invasiveness of oral squamous cell carcinoma through regulating the miR-637/NUPR1 axis. Biomed Pharmacother. 2019;120:109507.

32. Guan EC, Xu XG, Xue FX. circ-NOTCH1 acts as a sponge of miR-637 and affects the expression of its target gene Apelin to regulate gastric cancer cell growth. Biochem Cell Biol. 2019.

33. Li X, He M, Guo J, Cao T. Upregulation of circular RNA circ-ERBB2 predicts unfavorable prognosis and facilitates the progression of gastric cancer via miR-503/CACUL1 and miR-637/MMP19 signaling. Biochem Biophys Res Commun. 2019;511(4):926-30.

34. Li Y, Zhou L, Lu C, Shen Q, Su Y, Zhi Z, et al. Long non-coding RNA FAL1 functions as a ceRNA to antagonize the effect of miR-637 on the down-regulation of AKT1 in Hirschsprung's disease. Cell Prolif. 2018;51(5):e12489.

35. Arbelaiz A, Azkargorta M, Krawczyk M, Santos-Laso A, Lapitz A, Perugorria MJ, et al. Serum extracellular vesicles contain protein biomarkers for primary sclerosing cholangitis and cholangiocarcinoma. Hepatology. 2017;66(4):1125-43.

36. Macias RIR, Kornek M, Rodrigues PM, Paiva NA, Castro RE, Urban S, et al. Diagnostic and prognostic biomarkers in cholangiocarcinoma. Liver Int. 2019;39 Suppl 1:108-22.

37. Bergeat D, Fautrel A, Turlin B, Merdrignac A, Rayar M, Boudjema K, et al. Impact of stroma LOXL2 overexpression on the prognosis of intrahepatic cholangiocarcinoma. J Surg Res.

2016;203(2):441-50.

38. Sulpice L, Rayar M, Turlin B, Boucher E, Bellaud P, Desille M, et al. Epithelial cell adhesion molecule is a prognosis marker for intrahepatic cholangiocarcinoma. J Surg Res. 2014;192(1):117-23. 


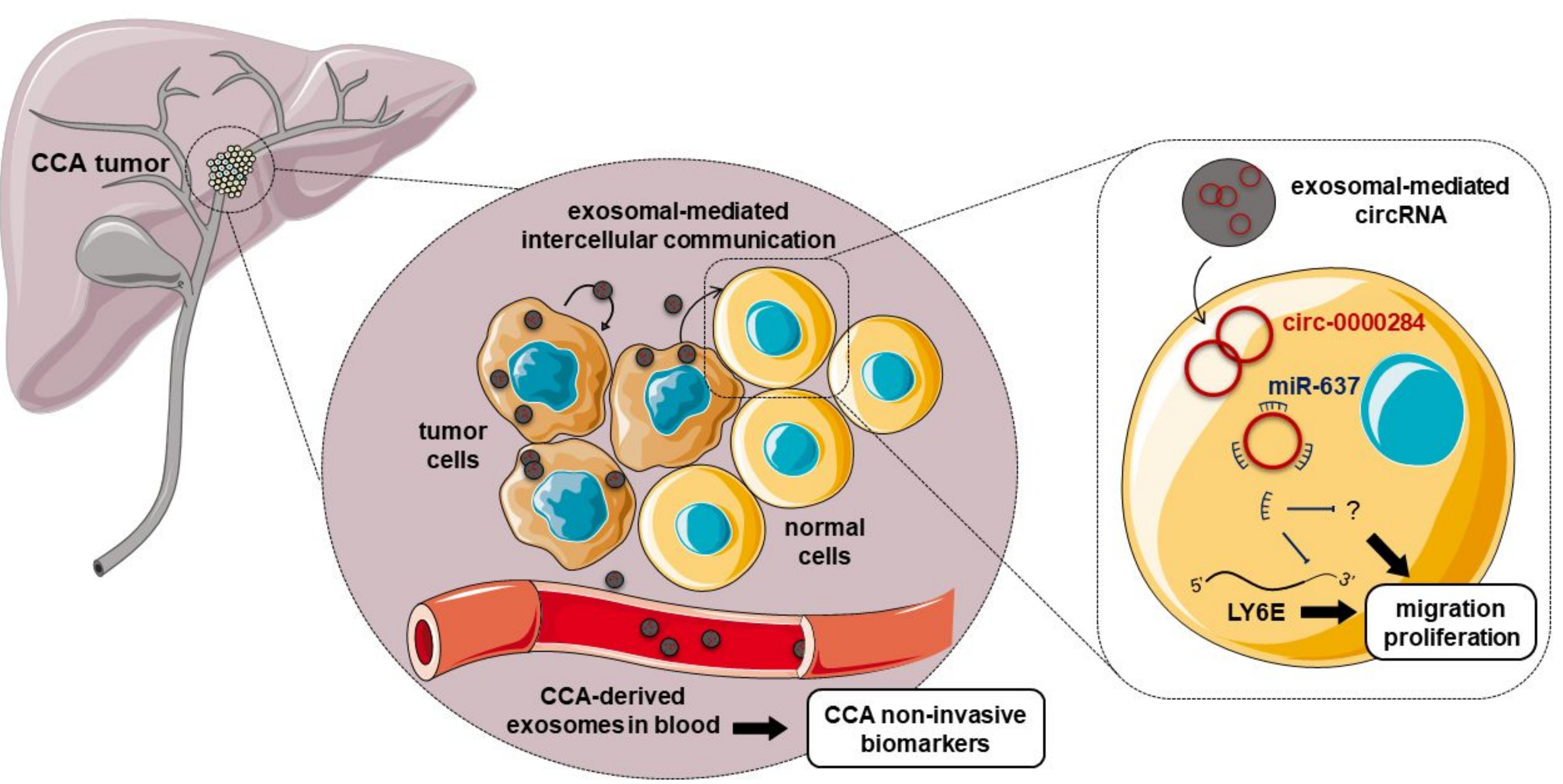


Table 1: Examples of cancer-specific role and regulatory functions of circ-000284

\begin{tabular}{|c|c|c|c|}
\hline Cancer type & Function & $\begin{array}{c}\text { Oncogene or Tumor } \\
\text { suppressor }\end{array}$ & Reference \\
\hline Bladder cancer & miRNA sponge $($ miR-558) & Tumor supressor & {$[23]$} \\
\hline Colorectal cancer & miRNA sponge (miR-7) & Oncogene & {$[24]$} \\
\hline $\begin{array}{c}\text { Hepatocellular } \\
\text { carcinoma }\end{array}$ & miRNA sponge (miR-124) & Oncogene & {$[20]$} \\
\hline Glioma & miRNA sponge (miR-654) & Oncogene & {$[21]$} \\
\hline $\begin{array}{c}\text { Nasopharyngal } \\
\text { carcinoma }\end{array}$ & miRNA sponge $($ miR-4288) & Oncogene & {$[22]$} \\
\hline Cholangiocarcinoma & miRNA sponge (miR-637) & Oncogene & {$[16]$} \\
\hline
\end{tabular}


Table 2: Examples of disease-specific targets and regulatory functions of miR-637.

\begin{tabular}{|c|c|c|c|}
\hline miR-637 target & $\begin{array}{l}\text { Endogenous miR-637 } \\
\text { competitor }\end{array}$ & Disease & Reference \\
\hline CDK6 & LINC00473 & Glioma & [26] \\
\hline KLK4 & circCEACAM5 & Ovarian cancer & [27] \\
\hline RING1 & C5orf66-AS1 IncRNA & Cervical cancer & [28] \\
\hline STAT3 & circHIPK3 & \multirow{2}{*}{ Colorectal cancer } & [29] \\
\hline \multirow{2}{*}{ NUPR1 } & FAL1 IncRNA & & {$[30]$} \\
\hline & LINC01234 & $\begin{array}{l}\text { Oral squamous cell } \\
\text { carcinoma }\end{array}$ & [31] \\
\hline APELIN & circNOTCH1 & \multirow{2}{*}{ Gastric cancer } & [32] \\
\hline MMP19 & circERBB2 & & [33] \\
\hline AKT1 & FAL1 IncRNA & Hirschsprung's disease & [34] \\
\hline
\end{tabular}

